\title{
Investigation of Membrane Mechanics using Spring Networks: Application to Red-blood-cell Modelling
}

\author{
Mingzhu Chen \\ Technological University Dublin, 452413@tudublin.ie \\ Fergal Boyle \\ Technological University Dublin, fergal.boyle@tudublin.ie
}

Follow this and additional works at: https://arrow.tudublin.ie/engschmecart

Part of the Biomaterials Commons, and the Molecular, Cellular, and Tissue Engineering Commons

\author{
Recommended Citation \\ doi:10.1016/j.msec.2014.07.043 \\ This Article is brought to you for free and open access by \\ the School of Mechanical and Design Engineering at \\ ARROW@TU Dublin. It has been accepted for inclusion in \\ Articles by an authorized administrator of ARROW@TU \\ Dublin. For more information, please contact \\ arrow.admin@tudublin.ie, aisling.coyne@tudublin.ie, \\ gerard.connolly@tudublin.ie. \\ Funder: Technological University Dublin
}

Chen, M., Boyle, F. : Investigation of membrane mechanics using spring networks: application to redblood-cell modelling. Materials Science and Engineering: C, vol. 43, 1 October 2014, p. 506-516.

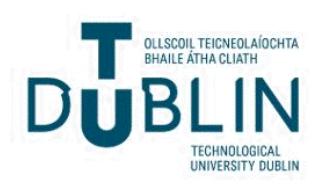




\title{
Investigation of membrane mechanics using spring networks: Application to red-blood-cell modelling
}

\author{
Mingzhu Chen ${ }^{*}$, Fergal J. Boyle ${ }^{1}$ \\ Department of Mechanical Engineering, School of Mechanical and Design Engineering, Dublin Institute of Technology, Bolton Street, Dublin 1, Ireland
}

\section{A R T I C L E I N F O}

\section{Article history:}

Received 10 March 2014

Received in revised form 11 June 2014

Accepted 13 July 2014

Available online 19 July 2014

\section{Keywords:}

Red blood cell

Membrane mechanics

Spring network

Discrete membrane

Network elasticity

Optical tweezers

\begin{abstract}
A B S T R A C T
In recent years a number of red-blood-cell ( $\mathrm{RBC}$ ) models have been proposed using spring networks to represent the RBC membrane. Some results predicted by these models agree well with experimental measurements. However, the suitability of these membrane models has been questioned. The RBC membrane, like a continuum membrane, is mechanically isotropic throughout its surface, but the mechanical properties of a spring network vary on the network surface and change with deformation. In this work spring-network mechanics are investigated in large deformation for the first time via an assessment of the effect of network parameters, i.e. network mesh, spring type and surface constraint. It is found that a spring network is conditionally equivalent to a continuum membrane. In addition, spring networks are employed for RBC modelling to replicate the optical tweezers test. It is found that a spring network is sufficient for modelling the RBC membrane but strain-hardening springs are required. Moreover, the deformation profile of a spring network is presented for the first time via the degree of shear. It is found that spring-network deformation approaches continuous as the mesh density increases.

(c) 2014 Elsevier B.V. All rights reserved.
\end{abstract}

\section{Introduction}

Blood is a suspension of cellular elements, including red blood cells (RBCs), white blood cells and platelets. RBCs dominate in terms of population with $5 \times 10^{6} / \mathrm{mm}^{3}$ and account for $99 \%$ of all suspended elements. A healthy RBC is biconcave in shape with a diameter of $8 \mu \mathrm{m}$ and a thickness of $2 \mu \mathrm{m}$. Structurally, a RBC may be considered as a liquid-core membrane-bounded capsule. The liquid, known as cytoplasm, is generally considered as an incompressible Newtonian fluid. The membrane has a dual-layer structure consisting of a plasma membrane and a cytoskeleton. The plasma membrane is a continuous layer mainly formed by a lipid bilayer while the cytoskeleton is a mesh-like elastic network. Mechanically, the plasma membrane is mainly responsible for the membrane surface incompressibility, bending and viscosity and the cytoskeleton mainly for the in-plane membrane shearing.

Due to the desire to understand microcirculation haemodynamics and RBC disorders, an increasing interest has been shown in RBC mechanics. However, an experiment-based study of RBC mechanics is usually not feasible because of the small RBC dimensions. Thus, numerical modelling emerges as a good alternative. The difficulty with RBC modelling is the accurate representation of the membrane mechanics. Currently, most membrane models are either based on continuum constitutive laws (continuum membrane models) or spring networks (discrete membrane models). A number of continuum membrane

\footnotetext{
* Corresponding author. Tel.: + 35314023963.

E-mail addresses: 452413@dit.ie (M. Chen), fergal.boyle@dit.ie (F.J. Boyle).

1 Tel.: + 35314023813
}

models have been proposed for the RBC membrane since the 1970s, e.g. Skalak membrane [1] and neo-Hookean membrane [2]. A continuum membrane model assumes the homogeneity of mechanical properties throughout the membrane surface. Due to the well-established theory of continuum mechanics, continuum RBC models are well developed for the study of cell mechanics [3-5], haemodynamics [6-8] and RBC disorders [9]. Discrete membrane models became popular in the late 1990s $[10,11]$ due to their simplicity and similarity to the cytoskeleton. A number of discrete RBC models yield a good representation of the cell mechanics [12] and capture the characteristics of the cell's response in flow [13-16]. However, discrete RBC models have been criticised for their anisotropic membrane properties $[17,18]$, i.e. spring-network properties.

The study of spring-network mechanics is well reviewed in a number of papers. Gelder [19] showed that a spring network cannot represent a continuum membrane model exactly, but a spring network can accurately represent an isotropic continuum membrane in small deformation if the spring constant is appropriately modified. Hansen et al. reviewed the mechanics of unstructured spring networks [20] and the impact of the networks' topology on their mechanical properties [21] However, both studies were restricted to small deformation and linear-spring types. Delingette [22] proposed an unusual spring type, i.e. a bi-quadratic spring which includes tensile and angular stiffness, for spring-network simulation. This new spring type showed the great potential of spring networks for accurate 2D membrane and 3D solid simulations [23]. Omori et al. [18] performed a comparison between discrete and continuum membrane models both for 2D and 3D applications. The paper made the important conclusion that a spring network is 
mechanically anisotropic and strain-softening, and thus is not suitable for modelling strain-hardening material, e.g. the RBC membrane.

The objective of this work is to investigate spring-network mechanics, and in particular the effect of network parameters which is frequently neglected in membrane modelling using spring networks. Critically, the suitability of a spring network for RBC modelling is examined. In Section 2, the effect of the network parameters on the mechanics of planar spring networks is investigated. In this work four spring element types are employed to construct spring networks: linear, truss, neo-Hookean (NH) [24] and worm-like-chains (WLC) [11]. In Section 3, these spring networks are used to construct discrete RBC models. The models are subsequently employed to replicate the optical tweezers (OT) test. In this work, membrane fluidity (viscosity) is ignored as we are interested only in the equilibrium state of deformation. Also, the continuum constitutive laws for the continuum membrane models are not discussed in detail here; a detailed description can be found in Barthès-Biesel et al. [25].

\section{Spring-network deformation and elasticity}

In this section the effect of network parameters, i.e. network mesh, spring element type and surface constraint, on springnetwork mechanics is investigated. Meanwhile, the network deformation is compared to deformation predicted using continuummembrane models, i.e. membrane models created using Hooke's Law (Hooke's), Mooney-Rivlin (MR) material [25] and Skalak (SK) Law [1]. First, a planar membrane is discretised to obtain a mesh representing a spring network with the lines considered the spring elements and the nodes the hinges. Since the membrane shape has no influence on the network properties [20], a square-shaped membrane is chosen. Having obtained a spring network, the networkrandomness metric [20] is introduced to measure the deviation of the network from an isotropic topology. Subsequently, the effect of the randomness and mesh density on the network elasticity is discussed. The elasticity of the network with a range of springelement types is predicted and compared to some continuum membrane models. Finally, the effect of surface constraint is also investigated.

\subsection{Construction of a 2D spring network}

A square-shaped membrane is discretised using the open-source mesh generator GMSH (GNU General Public License) to obtain an unstructured triangle-based mesh. As mentioned above the mesh represents the spring network with the lines the spring elements and the nodes the frictionless hinges. The network mesh is characterised by four topology parameters [20]: the average spring element length $\bar{L}$, the average node junction functionality $\bar{\varphi}$, i.e. the average number of springs connected to each node, the standard deviation of spring length $\sigma_{\mathrm{L}}$ and the standard deviation of node junction functionality $\sigma_{\varphi}$, see Fig. 1. Spring lengths are non-dimensionalised by dividing by the edge length of the discretised square. Therefore, the average spring length $\overline{\mathrm{L}}$ is a measure of mesh density of the network. $\sigma_{\mathrm{L}}$ is the standard deviation of the dimensionless spring length divided by $\overline{\mathrm{L}}$, so that $\sigma_{\mathrm{L}}$ is independent of the mesh density. $\sigma_{\mathrm{L}}$ and $\sigma_{\varphi}$ together are deemed the network randomness where $\sigma_{\mathrm{L}}$ is the length randomness and $\sigma_{\varphi}$ is the junction randomness. When the network randomness is zero, i.e. $\sigma_{\mathrm{L}}=0$ and $\sigma_{\varphi}=0$, the network consists of equilateral triangles, i.e. an isotropic topology. All networks employed in this work use an unstructured triangle-based mesh with high network randomness unless otherwise stated. An unstructured mesh is employed in this work for the following reasons:

1. A triangle-based mesh is the most stable topology for a spring network.

2. An isotropic mesh is not feasible for curved surfaces.

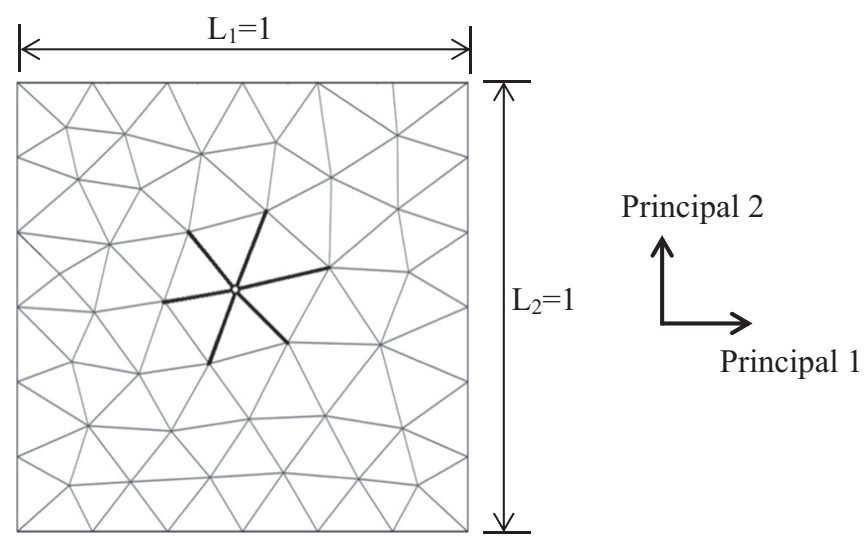

Fig. 1. A square-shaped membrane discretised with an unstructured mesh. All the element lengths are non-dimensionalised by dividing by the edge length; therefore, the square edges have a dimensionless length of one. The node (white dot) in the centre of the network has a junction functionality of six as six springs are connected to this node. For a given network the element connectivity is fixed, i.e. $\bar{\varphi}$ and $\sigma_{\varphi}$ are constant, but $\bar{L}$ and $\sigma_{L}$ vary as the network undergoes deformation. Note that in calculating $\bar{\varphi}$, the junction functionalities of the nodes on the boundary are not considered.

3. Among the triangle-based meshes, an unstructured mesh is the most popular in numerical simulations.

4. An unstructured mesh converges towards being isotropic as the network randomness decreases.

\subsection{Computation of spring-network deformation}

A spring network deforms upon external loading. This deformation is modelled by updating the nodes' position with time. The updating scheme employed is formed using the Taylor series expansion, i.e.

$p_{i}(t+\Delta t)=p_{i}(t)+\Delta t \times p_{i}^{\prime}(t)+\frac{\Delta t^{2}}{2} \times p_{i}^{\prime \prime}(t)+\frac{\Delta t^{3}}{6} \times p_{i}^{\prime \prime \prime}(t)+\ldots$

where $\mathrm{p}$ refers to the position of a node, superscript ' refers to the derivative with respect to time $t$, subscript $i$ refers to principal direction 1 or 2 , and $\Delta t$ is the time-step. Viscous damping has to be introduced in Eq. (1) or the spring network oscillates continually. Assuming that each node is associated with a damping which eliminates the node speed, i.e. $p_{i}^{\prime}(t)$, at each time step and that the high-order differential terms are neglected, Eq. (1) reduces to

$$
\begin{aligned}
p_{i}(t+\Delta t) \simeq p_{i}(t)+\frac{\Delta t^{2}}{2} \times p_{i}^{\prime \prime}(t)= & p_{i}(t)+\frac{\Delta t^{2}}{2} \\
& \times \frac{\sum F_{p_{i}(t)}}{m}=p_{i}(t)+\sum F_{p_{i}(t)} \times \Delta T(2)
\end{aligned}
$$

where $\sum \mathrm{F}$ is the total force acting on the node, $\mathrm{m}$ is the node mass and $\Delta \mathrm{T}$ is a constant formed by combining $\Delta \mathrm{t}$ and $\mathrm{m}$, i.e. $\Delta \mathrm{T}=\left(\Delta \mathrm{t}^{2}\right) /(2 \mathrm{~m})$. This modified equation cannot accurately predict the transient motion of a node, but is well suited for the calculation of an equilibrium position [18] subject to a convergence tolerance, e.g. $\left|\sum \mathrm{F}_{\mathrm{pi}(\mathrm{t})} \times \Delta \mathrm{T}\right|<10^{-8}$.

Four spring element types are employed in this work: linear, truss, $\mathrm{NH}$ and WLC representing linearly-elastic springs, linearly-elastic springs with varying spring constant, strain-softening springs, and non-linear strain-hardening springs respectively defined as follows:

$F_{\text {linear }}=-k d l$

$F_{\text {truss }}=-\frac{k}{l_{o}} d l$ 
$F_{N H}=-(\lambda-1) \frac{\lambda^{0.5}+\lambda^{-2.5}}{\lambda+\lambda^{-3}}$

$F_{W L C}=-\frac{k_{B} T}{p}\left[\frac{1}{4(1-x)^{2}}-\frac{1}{4}+x-C\right]$

where $\mathrm{F}$ is the force exerted from a spring to the nodes at its ends, $\mathrm{k}$ is the spring constant, 1 is the instantaneous length of the spring, $l_{0}$ is the initial length of the spring, $\lambda\left(=l / l_{o}\right)$ is the stretch ratio of the spring, $\mathrm{k}_{\mathrm{B}}$ is the Boltzmann constant, $\mathrm{T}$ is the temperature, $\mathrm{p}$ is the persistent length of WLC elements and $x\left(=l / l_{\text {contour }}\right)$ is the ratio of the instantaneous element length to the contour (maximum) length of the WLC. The WLC force equation differs from the conventional form as a constant $\mathrm{C}$ is introduced to give the spring an initial length [26]. The $\mathrm{C}$ value is defined as

$C=\frac{1}{4\left(1-x_{0}\right)^{2}}-\frac{1}{4}+x_{o}$

where $x_{o}\left(=l_{o} / l_{\text {contour }}=1 / 3.17\right)$ is the $\mathrm{x}$ at initial configuration as the WLC contour length is defined as $3.17 l_{\mathrm{o}}$. Importantly, the definition of the contour length manipulates the hardening effect of a WLC element.

A constraint on area dilation is introduced into a spring network via the dilation energy of each network triangle, i.e.

$F_{p, \text { i.dilation }}=\left.\frac{\partial V_{\text {area }}}{\partial i}\right|_{p}=-\left.\frac{\partial\left(\frac{K_{A}}{2 A_{o}}\left(A-A_{o}\right)^{2}\right)}{\partial i}\right|_{p}$

where $\mathrm{F}$ is the force exerted from the dilation resistance onto node $\mathrm{p}, \mathrm{V}_{\text {area }}$ is the strain energy stored in a triangle which contains node $\mathrm{p}$, $\mathrm{i}$ represents principal direction 1 or $2, \mathrm{~A}$ and $\mathrm{A}_{\mathrm{o}}$ are the current and the initial areas of the triangle respectively, and $\mathrm{K}_{\mathrm{A}}$ is the dilation modulus for a local-area-constraint (LAC) which resists the area dilation of each triangle. A global-area-constraint (GAC), on the other hand, resists the total surface area change of a spring network. Note that a LAC ensures a GAC, but a GAC does not ensure a LAC; also a GAC is equivalent to a LAC in $2 \mathrm{D}$ applications of spring networks. In our simulations $\mathrm{K}_{\mathrm{A}}=1,000,000$, so that a spring network almost conserves the area of each triangle.

\subsection{Computation of spring-network properties}

The properties associated with a membrane surface include shear modulus $\mu$, Young's modulus E, Poisson's ratio $\nu$ and area dilation modulus K. Membrane viscosity is neglected in this work since we are interested only in the equilibrium state of a spring network.

The derivation of the spring-network properties is briefly explained here; more details can be found in Hansen et al. [20]. Consider the deformation of a triangle-shape membrane modelled using a three-element spring network as shown in Fig. 2. The triangle strains $\varepsilon$ can be obtained as follows:

$$
\begin{aligned}
\left(l^{2}-l_{o}^{2}\right)_{p, q, r} & =\left(2 \varepsilon_{i j}\left(L_{i}\right)\left(L_{j}\right)\right)_{p, q, r} \\
& =\left(2 \varepsilon_{11}\left(L_{1}\right)^{2}+4 \varepsilon_{12}\left(L_{1}\right)\left(L_{2}\right)+2 \varepsilon_{22}\left(L_{2}\right)^{2}\right)_{p, q, r}
\end{aligned}
$$

where $l$ and $l_{o}$ are the deformed and initial lengths of a spring element, $\mathrm{L}$ is the component length of $l_{o}$ in principal direction 1 or 2 , and the subscripts of the strains $\varepsilon$ refer to the strain direction. Applying the above relationship to all three spring elements we have

$$
\left(\begin{array}{ccc}
2\left(L_{1}^{2}\right)_{p} & 4\left(L_{1} L_{2}\right)_{p} & 2\left(L_{2}^{2}\right)_{p} \\
2\left(L_{1}^{2}\right)_{q} & 4\left(L_{1} L_{2}\right)_{q} & 2\left(L_{2}^{2}\right)_{q} \\
2\left(L_{1}^{2}\right)_{r} & 4\left(L_{1} L_{2}\right)_{r} & 2\left(L_{2}^{2}\right)_{r}
\end{array}\right)\left(\begin{array}{c}
\varepsilon_{11} \\
\varepsilon_{12} \\
\varepsilon_{22}
\end{array}\right)=\left(\begin{array}{c}
\left(l^{2}-l_{o}^{2}\right)_{p} \\
\left(l^{2}-l_{o}^{2}\right)_{q} \\
\left(l^{2}-l_{o}^{2}\right)_{r}
\end{array}\right) .
$$

Note the square matrix on the left hand side is known from the initial configuration which means that the triangle strains $\varepsilon_{11}, \varepsilon_{22}$ and $\varepsilon_{12}$ are functions of the lengths of the deformed springs. Also, the triangle principal stretch ratios $\lambda_{1}$ and $\lambda_{2}$ and the network Poisson's ratio $v$ are related to the principal strain components as follows

$\lambda_{1}=\sqrt{2 \varepsilon_{11}+1}$

$\lambda_{2}=\sqrt{2 \varepsilon_{22}+1}$

$\nu=-\frac{\varepsilon_{22}}{\varepsilon_{11}}$.

The triangle strain energy density accounts for the strain energies of all of the springs of the network, i.e.

$V=\frac{1}{A_{t}} \sum^{n}\left(\int_{l_{0}}^{l} F_{\text {spring }} d l\right)$

where $V$ is the energy density of the spring network, $A_{t}$ is the network area in the initial configuration, and $n$ is the total number of spring elements. Using the energy density, the shear and dilation moduli of the spring network are calculated as follows

$\mu=\left(\frac{\partial V}{\partial \beta}\right)_{\alpha}$

$$
K=\left(\frac{\partial^{2} V}{\partial \alpha^{2}}\right)_{\beta}
$$
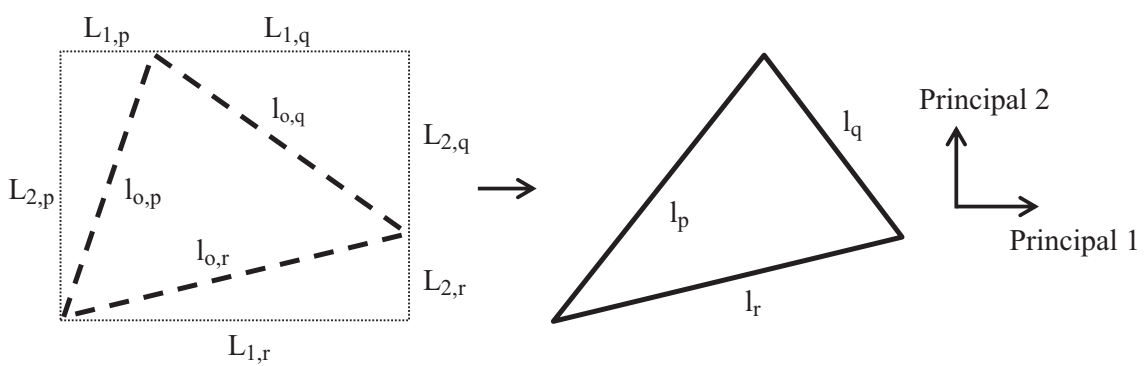

Fig. 2. Deformation of a triangle consisting of edges p, q and r [20]. The broken and solid lines are the boundaries of the initial and deformed triangles respectively. 
where $\alpha$ is the fractional area dilation and $\beta$ is the degree of shearing from a circle to an ellipse, defined as

$\alpha=\lambda_{1} \lambda_{2}-1$

$\beta=\frac{\left(\lambda_{1}-\lambda_{2}\right)^{2}}{2 \lambda_{1} \lambda_{2}}$

\subsection{Elasticity of a spring network}

Hansen et al. [20] showed that network randomness measures the departure of spring-network properties from that of an isotropic network. Also, the network properties vary upon deformation. Therefore, a discussion of spring-network properties is complex and insufficient to demonstrate the network mechanics. In this work the springnetwork, i.e. discrete membrane model, mechanics are investigated through the network elasticity [18], which is measured by the slope of tension-strain and tension-dilation curves of the membrane under uniaxial and isotropic loadings respectively. Uniaxial deformation is a combination of shearing and dilation while isotropic deformation is solely due to dilation, see Fig. 3. In other words, the slope of tension-strain curve measures a combination of the shear and dilation moduli while the slope of tension-dilation curve directly measures the dilation modulus [5]. As a result the network elasticity is adequate to represent the overall network mechanics. Also, tension in the tension-strain and tension-dilation curves is non-dimensionalised by dividing by the initial shear modulus so that the effect from the initial spring constant and mesh density is eliminated.

\subsubsection{Effect of network randomness on the direction dependence of the network elasticity}

Since the network randomness measures the departure of a network's properties from that of an isotropic network, the effect of randomness on the network elasticity is examined. Fig. 4 shows the theoretical tension-strain relationship of an isotropic linear-spring network for two uniaxial loading scenarios (the derivation of the theoretical elasticity is given in the Appendix). The results indicate the elasticity of an isotropic network depends on the direction of loading. Along with the elasticity under uniaxial deformation, the isotropic network shear modulus is also direction dependent [27] in large deformation.

Fig. 5 shows the predicted tension-strain relationship of two linearspring networks under uniaxial loading. Both networks have a similar network density $\overline{\mathrm{L}} \approx 0.018$. The high-randomness network has $\sigma_{L}=0.15$ and $\sigma_{\varphi}=0.87$, while the low-randomness network has $\sigma_{L}=0.038$ and $\sigma_{\varphi}=0.23$. From observation, the curves for the network with the higher randomness are almost overlapping each other on the measuring range. Curves for the low-randomness network deviate slightly up to $100 \%$ strain and the direction dependence of the elasticity is pronounced when the strain is greater than $100 \%$. The

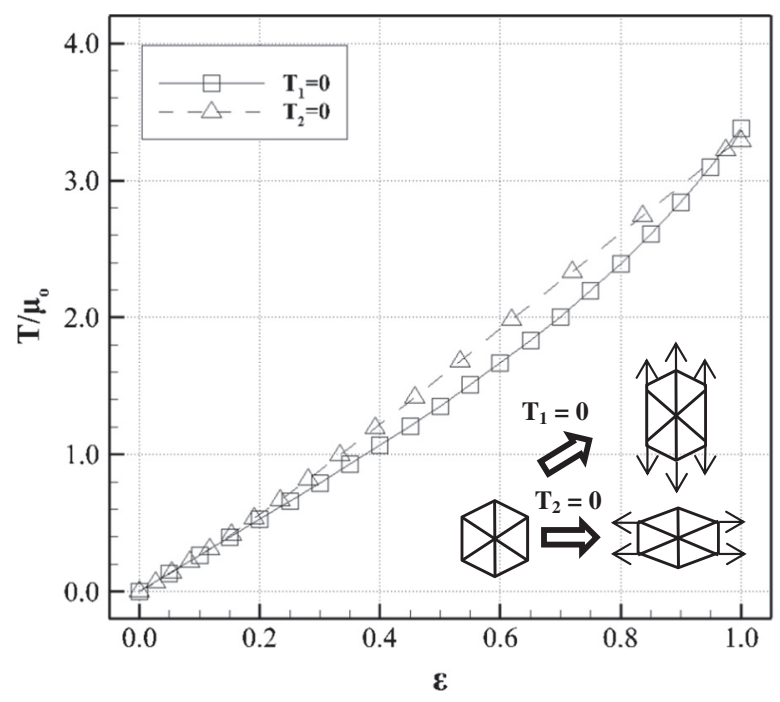

Fig. 4. Theoretical tension-strain relationship of an isotropic linear-spring network with different loading directions. The loading follows two uniaxial directions as indicated.

direction dependence of the network elasticity is caused by the unequal contribution of the spring elements in both principal directions. A high randomness increases the probability of the equal contribution of the spring elements in both principal directions and therefore the direction dependence of network elasticity is greatly reduced. Fig. 4 shows that an isotropic spring network is mechanically isotropic in small deformation while Fig. 5 shows that a spring network with high randomness tend to be mechanically isotropic even in large deformation.

\subsubsection{Effect of mesh density on network elasticity}

The foregoing simulation results suggest that high network randomness eliminates the direction dependence of the network elasticity. Here the effect of the mesh density on the network elasticity is examined. Fig. 6 shows the predicted tension-strain relationship of four linear-spring networks under uniaxial loading. The dimensionless average lengths $\bar{L}$ of the four networks are 0.0094, 0.0189, 0.0994 and 0.2487 . Also, the four networks have similar high randomness, i.e. $0.14<\sigma_{\mathrm{L}}<0.18,0.8<\sigma_{\varphi}<1.1$. The results show that the four networks have almost identical network elasticity which indicates that the network elasticity is independent of the network mesh density.

\subsubsection{Effect of spring element type on network elasticity}

Previously, the effect of the network randomness and the mesh density on the network elasticity was investigated. Here, four spring types are employed in a network to predict network elasticity under uniaxial and isotropic loadings. The network has parameters of $\overline{\mathrm{L}}=0.0189, \bar{\varphi}=5.8$, $\sigma_{L}=0.15$ and $\sigma_{\varphi}=0.95$. Fig. 7 shows the predicted network elasticity using the different spring types along with the analytical

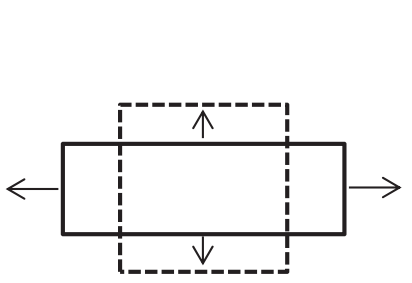

(a)

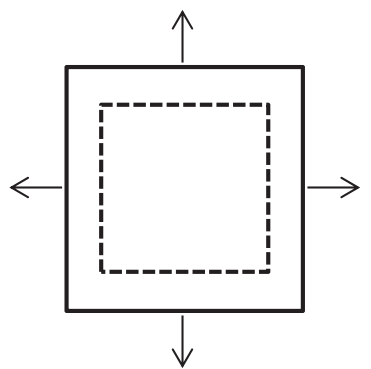

(b)
Principal 2

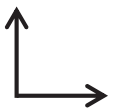

Principal 1

Fig. 3. (a) Uniaxial and (b) isotropic deformations of a 2D membrane. The broken and solid lines refer to the initial and deformed geometries respectively. 


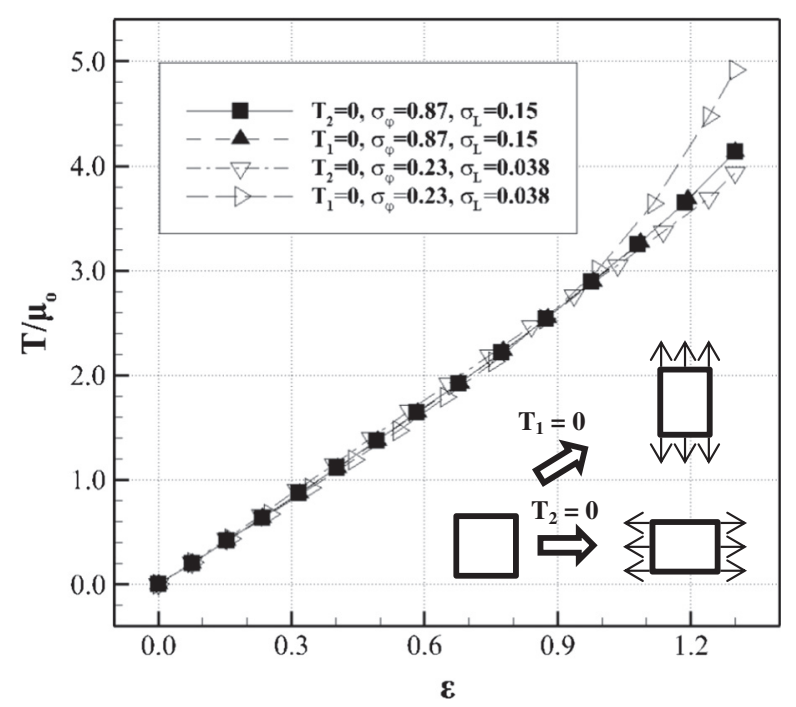

Fig. 5. Predicted tension-strain relationship of two linear-spring networks with different network randomness. The loading follows two uniaxial directions as indicated.

membrane elasticity predicted using continuum-membrane models. Fig. 7(a) indicates that the WLC network is strain hardening as well as the SK membrane. As the WLC spring is strain-hardening, i.e. the spring constant increases as the strain increases, therefore, the network resistance increases as the strain increases. On the other hand, the $\mathrm{NH}$ network is strain-softening as the spring constant of the elements decreases as the strain increases. The tension-strain relationships of the linear-spring and truss networks are similar to the continuum membrane model based on Hooke's law. The truss network is slightly stiffer than the linear-spring network because the initial shear modulus of the truss network is slightly smaller than that of the linear-spring network. Fig. 7(b) shows that all spring networks are vulnerable to surface dilation. The results agree with the prediction by Omori et al. [18] that the surface area of a spring network dilates twice as fast as the dilation force in an isotropic loading test, i.e. $d S / d T \approx 2$. Therefore, all spring networks are naturally vulnerable against surface area dilation regardless of the spring type. The results show the behaviour of spring constant defines the spring network elasticity in large deformation,

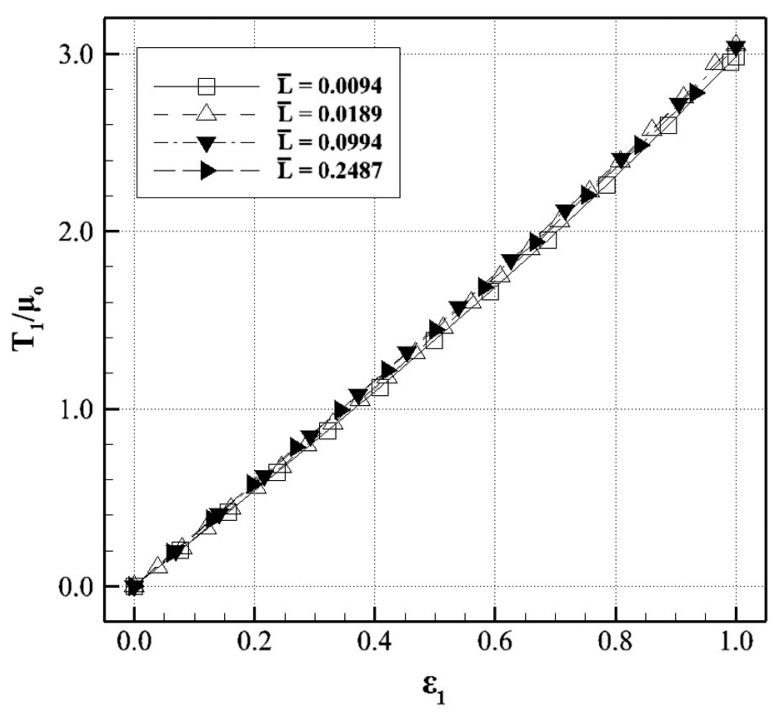

Fig. 6. Predicted tension-strain relationship of four linear-spring networks with different mesh densities. The loading direction is the same for the four networks, i.e. $T_{2}=0$. (a)

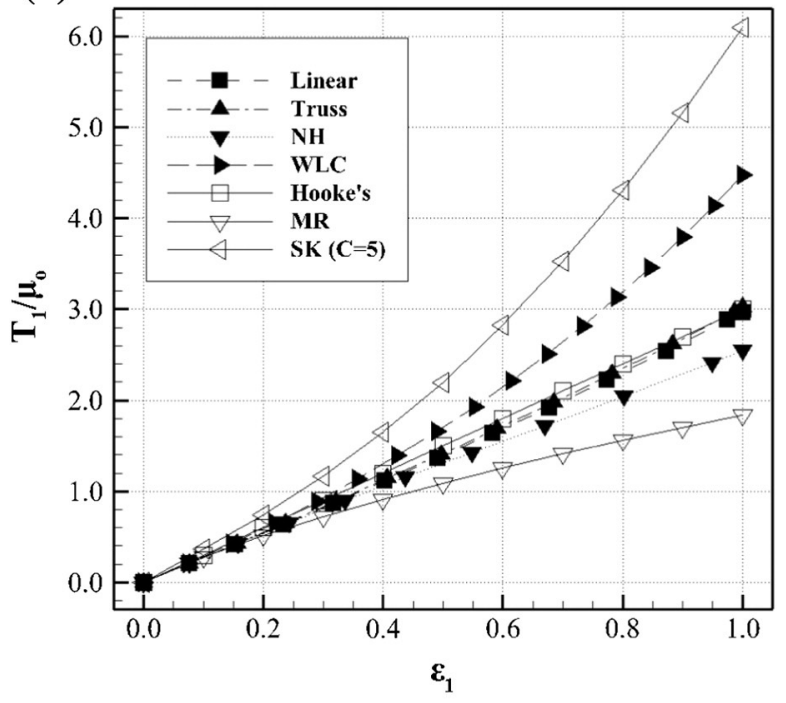

(b)

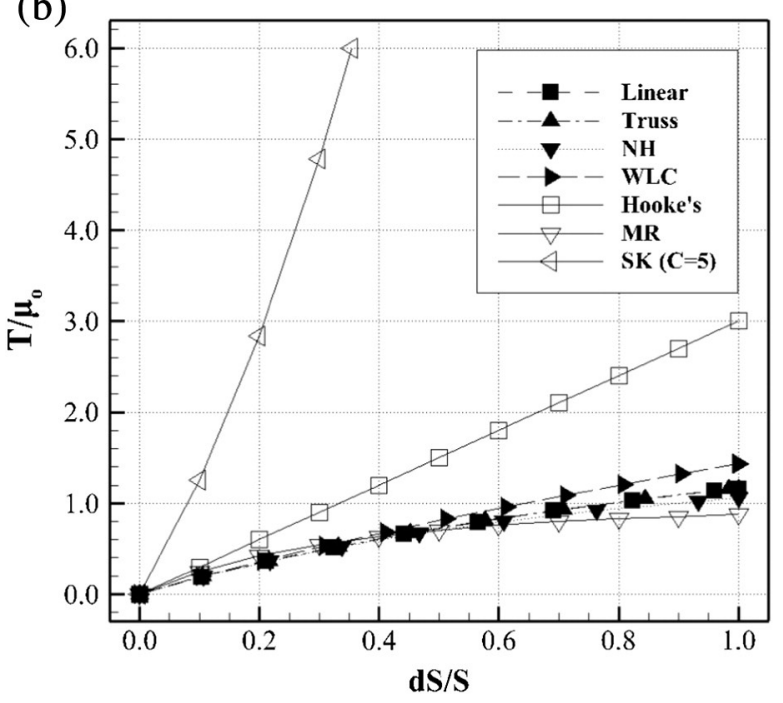

Fig. 7. Predicted tension-strain relationship of membrane models under (a) uniaxial loading and (b) isotropic loading. dS is the change in surface area and S is the initial surface area of the membrane models. The curves with filled symbols are predicted results for spring networks with the spring types indicated in the legend; the curves with empty symbols are the analytical results for continuum membrane models with the continuum constitutive laws indicated in the legend.

e.g. an increase in the spring constant leads to a hardening-effect of the spring network and vice versa.

Fig. 8(a) and (b) shows the degree of shear, i.e. $\beta$ in Eq. (18), of the spring networks under uniaxial loading. Fig. 8(a) is for the surface-unconstrained networks with $\varepsilon_{1} \approx 1.02$ and (b) is for the surface-constrained networks with $\varepsilon_{1} \approx 1.08$. Fig. $8(\mathrm{c})$ shows the fractional area dilation, i.e. $\alpha$ in Eq. (17), under isotropic loading with $\mathrm{dS} / \mathrm{S} \approx 0.96$. The results first indicate the variation of local network mechanics which is due to the variation of local network randomness. Second, the degree of variation differs from one spring network to the other, e.g. the variation in the $\mathrm{NH}$ network is the largest while the variation in the WLC network is the smallest. Third, and importantly, regardless of the local variation, the spring networks deform homogeneously as the loading is applied which suggests that their deformation is equivalent to that of continuum membrane models. Small deviations are observable at the boundary edges which are due to the Saint-Venant principle [18] and vanish as the mesh is fined. 

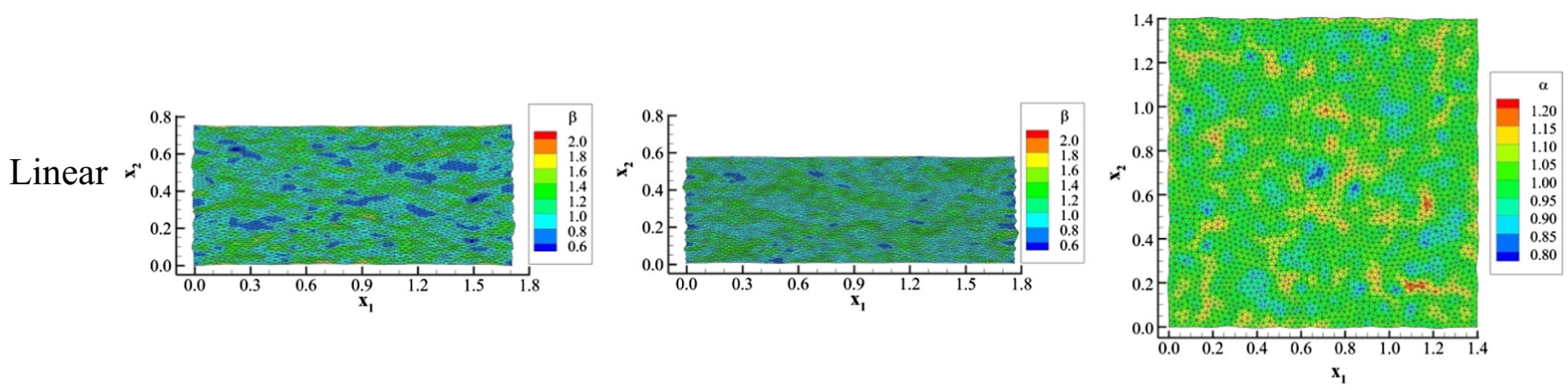

Truss
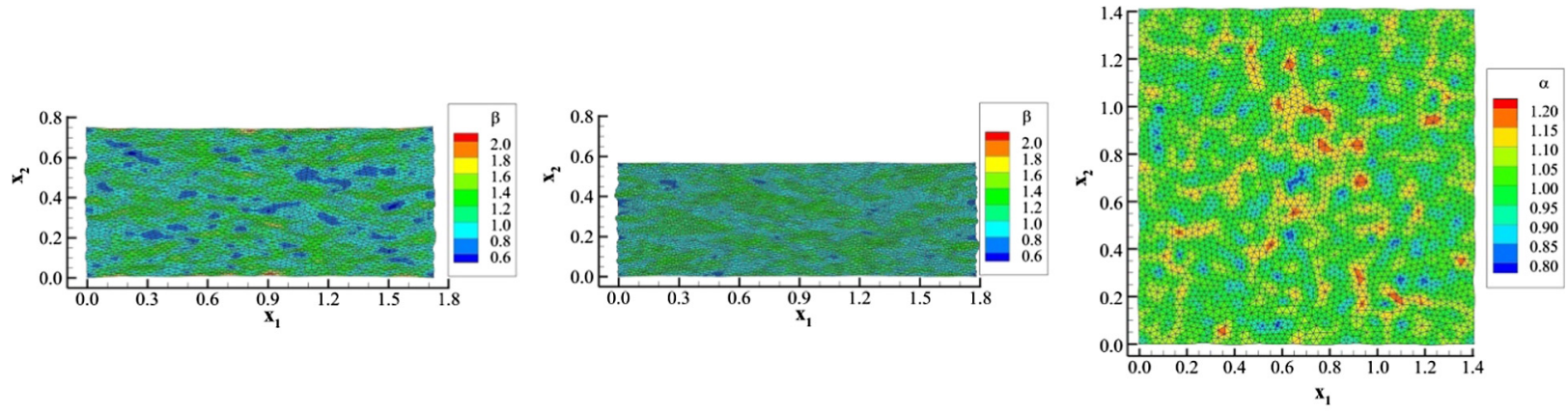

$\mathrm{NH}$
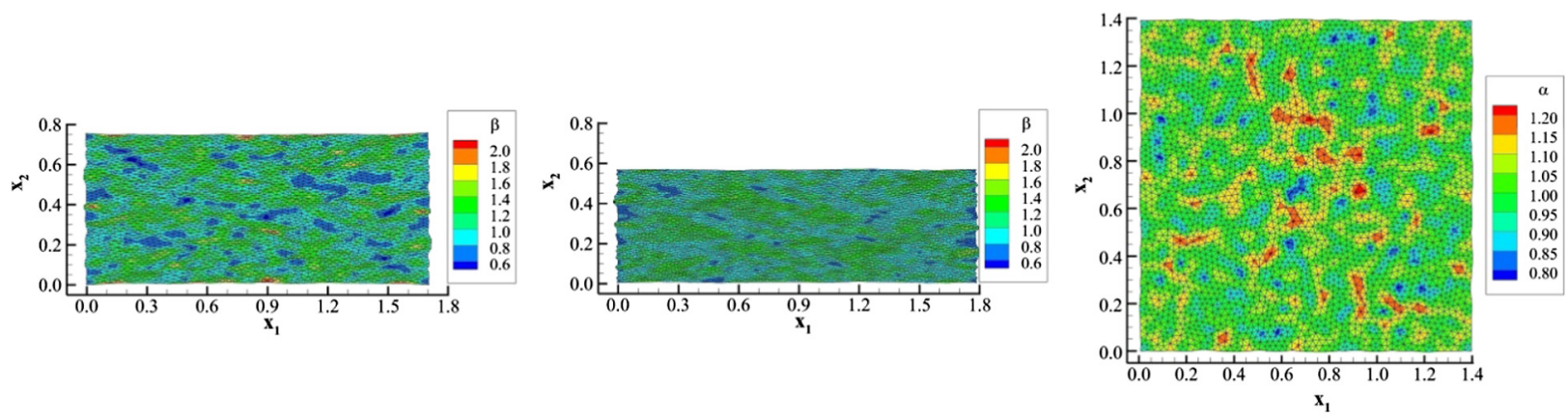

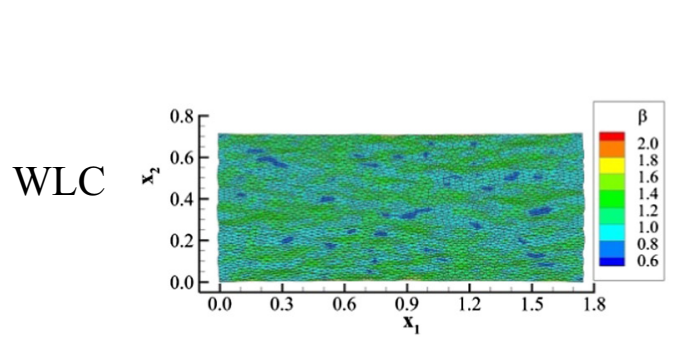

(a)

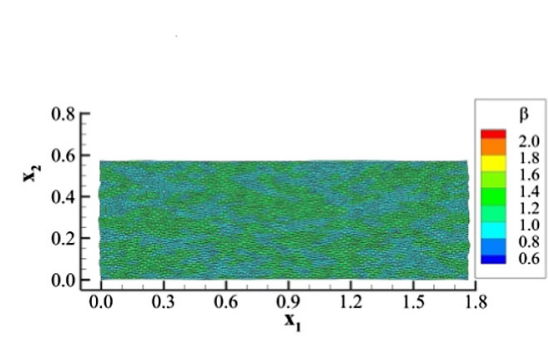

(b)

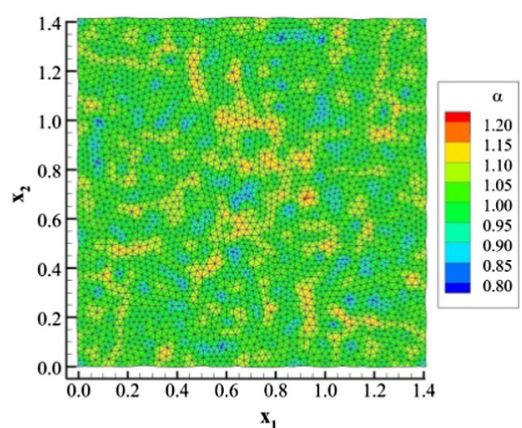

(c)

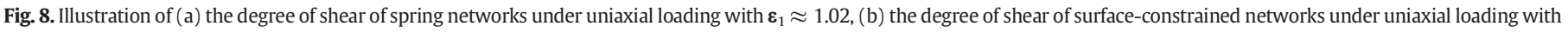
$\boldsymbol{\varepsilon}_{1} \approx 1.08$ and (c) the fractional area dilation under isotropic loading with $\mathrm{dS} / \mathrm{S} \approx 0.96$.

\subsubsection{Effect of surface constraint on network elasticity}

The results in Figs. 7 and 8 indicate that the spring networks have shear moduli comparable to continuum membrane models and that the spring networks have small dilation moduli. Next, network elasticity is predicted for the spring networks when a surface constraint is enforced. Delingette [22] introduced such a constraint via spring angular stiffness which restricts the rotation of spring elements. For numerical convenience, in this work a LAC is introduced into the spring networks. The tension-strain relationship of the spring networks with a LAC under uniaxial loading is predicted. The results are compared to those of continuum membrane models in Fig. 9. The results indicate that the elasticity of the spring networks reduces significantly when the surface constraint is enforced. Also, the WLC-spring network becomes comparable to the SK membrane $(C=5)$. When the surface constraint is not applied, the network deformation orientates spring elements inline to the tension and creates strains and dilations. When the constraint is applied, the deformation is resisted by compensating isotropic tensions on each triangle which reduces the network elasticity. Additionally, the isotropic compensation yields continuity to the spring networks which leads to a more uniform distribution of network properties, e.g. the degree of shear in surface-constrained networks is a lot more uniform than that in the non-constrained spring networks, see 


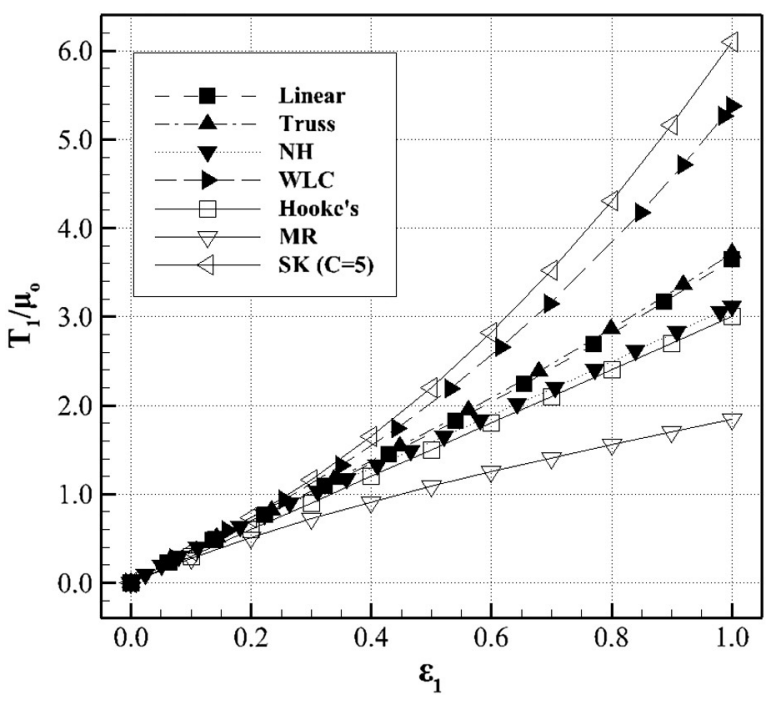

Fig. 9. Predicted tension-strain relationship of membrane models under uniaxial loading. Note the spring networks are area-dilation constrained.

Fig. 8(a) and (b). Finally, note that isotropic loading tests are not available for the spring networks with a LAC, since the LAC dilation modulus is so large that the spring networks resist any surface area dilation; thus the tension for isotropic deformation is infinity.

\section{Discrete RBC modelling and deformation}

The RBC membrane is a strain-hardening material [28]. It has a thickness on the nano-metre scale, three orders smaller than the size of the cell. Thus, a membrane model is usually assumed to have a negligible thickness. Here the suitability of a spring network for RBC membrane modelling is examined. In this work a biconcave solid is first created and discretised using commercial packages. The discretised surface mesh forms the network for our models. Four spring types are employed to construct discrete RBC models using the network. The models are subsequently used to replicate the OT test. The predicted results are compared to experimental measurements [4]. In addition, the effect of the spring-element type and mesh density on RBC modelling is discussed. Measured RBC membrane properties vary significantly in a number of experimental measurements. For example, the bending modulus ranges from 0.2 to $7 \times 10^{-19} \mathrm{Nm}$ and the shear modulus ranges from 1 to $13 \mu \mathrm{N} / \mathrm{m}$ [3]. The commonly accepted values of bending modulus and shear modulus are $2.5 \times 10^{-19} \mathrm{Nm}$ and $4.8 \mu \mathrm{N} / \mathrm{m}$ respectively. In our simulations the network bending modulus $\mathrm{K}_{\text {bend }}$ is set as $2.5 \times 10^{-19} \mathrm{Nm}$. Also, the spring constant is adjusted so that the corresponding isotropic spring network yields a shear modulus $\mu_{0}$ of $4.5 \mu \mathrm{N} / \mathrm{m}$. However, the shear modulus of the spring-network RBC membrane is slightly larger than $4.5 \mu \mathrm{N} / \mathrm{m}$ because the 3D network topology is anisotropic with non-zero network randomness.

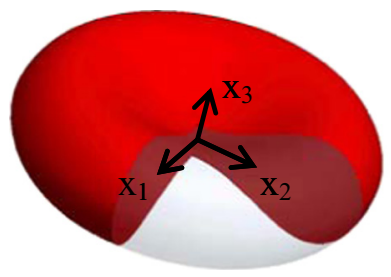

(a)

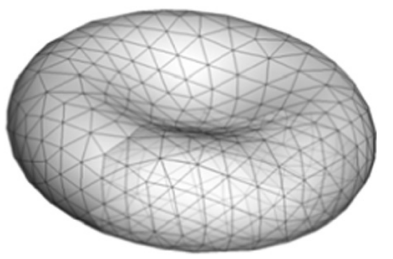

(b)
Fig. 10. A RBC solid model (a) discretised to give a network on the surface (b).

\subsection{Construction of the 3D RBC model}

The shape of an average undeformed RBC surface may be represented by the following equation [29]

$x_{3}= \pm D_{o}\left(1-\frac{4\left(x_{1}^{2}+x_{2}^{2}\right)}{D_{o}^{2}}\right)^{1 / 2}\left(a_{0}+a_{1} \frac{x_{1}^{2}+x_{2}^{2}}{D_{o}^{2}}+a_{2} \frac{\left(x_{1}^{2}+x_{2}^{2}\right)^{2}}{D_{o}^{4}}\right)$

where $\mathrm{x}$ is the coordinate value and subscripts 1,2 and 3 represent the principal directions. $D_{0}$ is the initial diameter of the $R B C$ with a value of $7.82 \mu \mathrm{m}$, and $\mathrm{a}_{0}, \mathrm{a}_{1}$ and $\mathrm{a}_{2}$ are constants with values of $0.0518,2.026$ and -4.491 respectively. This equation is employed in SolidWorks (SolidWorks Corp. Massachusetts, U.S.) to obtain a 3D solid. The solid is subsequently discretised using Ansys (ANSYS, Inc., Canonsburg, U.S.) to obtain a surface network, see Fig. 10. Three surface-network meshes, with details listed in Table 1, are employed for the construction of RBC models. Mesh 2 is used for the RBC modelling unless otherwise stated.

\subsection{Computation of RBC mechanics}

Besides the viscosity, the properties associated with a RBC are the membrane shear modulus, bending modulus, surface area incompressibility and volume incompressibility. For numerical convenience, the membrane bending resistance is derived from the instantaneous angle between neighbouring triangles with reference to the spontaneous angle as in Fig. 11 [30], i.e.

$F_{p 1 . \text { bend }}=-K_{B} \tan \left(\frac{\theta-\theta_{0}}{2}\right) \mathbf{n}_{123}$

$F_{p 4 . b e n d}=-K_{B} \tan \left(\frac{\theta-\theta_{0}}{2}\right) \mathbf{n}_{234}$

$F_{p 2 . \text { bend }}=F_{p 3 . \text { bend }}=-\frac{F_{p 1}+F_{p 4}}{2}$

where $\mathrm{F}$ is the force exerted from the bending resistance onto node $\mathrm{p}, \mathrm{K}_{\mathrm{B}}$ is the bending stiffness defined as $K_{B}=(2 / \sqrt{3}) K_{\text {bend }}[11,17], \theta$ and $\theta_{\mathrm{o}}$ are the instantaneous and spontaneous angles respectively between two neighbouring triangles, and $\mathbf{n}_{\mathbf{i j k}}$ is the normal vector of the triangle consisting of nodes $\mathrm{p}_{\mathrm{i}}, \mathrm{p}_{\mathrm{j}}$ and $\mathrm{p}_{\mathrm{k}}$.

Similar to the surface constraint in a 2D spring network, the surface area and volume constraints in the RBC model are introduced via the energy function, i.e.

$$
\begin{aligned}
F_{p, \text { i.area }} & =-\left.\frac{\partial V_{\text {local.area }}+\partial V_{\text {global.area }}}{\partial i}\right|_{p} \\
& =-\left.\frac{\partial\left(\frac{K_{A}}{2 A_{o}}\left(A-A_{o}\right)^{2}+\frac{K_{A}^{\text {total }}}{2 A_{o}^{\text {total }}}\left(A^{\text {total }}-A_{o}^{\text {total }}\right)^{2}\right)}{\partial i}\right|_{p}
\end{aligned}
$$

$F_{p, i \text {.volume }}=-\left.\frac{\partial V_{\text {global. volume }}}{\partial i}\right|_{p}=-\left.\frac{\partial\left(\frac{K_{t}^{\text {total }}}{2 V_{o}^{\text {total }}}\left(V^{\text {total }}-V_{o}^{\text {total }}\right)^{2}\right)}{\partial i}\right|_{p}$

Table 1

RBC network-meshes with network parameters. $N_{v}$ is the number of nodes in the network mesh and $\mathrm{N}_{\mathrm{s}}$ the number of spring elements.

\begin{tabular}{llrllll}
\hline Mesh & $\mathrm{N}_{\mathrm{v}}$ & \multicolumn{1}{c}{$\mathrm{N}_{\mathrm{s}}$} & $\overline{\mathrm{L}}(\mu \mathrm{m})$ & $\bar{\varphi}$ & $\sigma_{\mathrm{L}}(\mu \mathrm{m})$ & $\sigma_{\varphi}$ \\
\hline 1 & 6467 & 19,395 & 0.15 & 6.00 & 0.020 & 0.37 \\
2 & 1334 & 3996 & 0.34 & 5.99 & 0.034 & 0.41 \\
3 & 235 & 699 & 0.81 & 5.95 & 0.120 & 0.55 \\
\hline
\end{tabular}




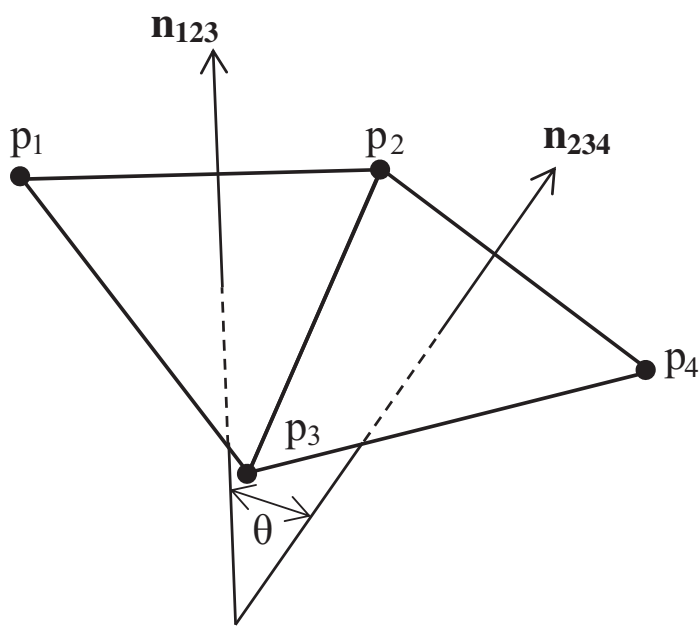

Fig. 11. Illustration of the angle between two neighbouring triangles used to calculate bending resistance.

where $F_{p, i, a r e a}$ and $F_{p, i, v o l u m e}$ are the forces on node $p$ in direction $i$ exerted from the area dilation and volume bulk resistance respectively, $\mathrm{V}$ is strain energy, $\mathrm{K}_{\mathrm{A}}$ is a LAC dilation modulus with a default value of $100 \mathrm{pN} / \mu \mathrm{m}^{2}, K_{A}^{\text {total }}$ is a GAC dilation modulus with a default value of $5000 \mathrm{pN} / \mu \mathrm{m}^{2}, K_{V}^{\text {total }}$ is the bulk constant with a default value of $5000 \mathrm{pN} / \mu^{3}{ }^{3}, A$ is the local area of the triangle which includes node $\mathrm{p}, \mathrm{A}^{\text {total }}$ is the total surface area of the RBC model, $\mathrm{V}^{\text {total }}$ is the volume of the RBC model, and the subscript o means that the value corresponds to the initial configuration. Physically, surface area conservation of a RBC consists of a LAC and a GAC. The LAC is due to the movement restriction of the anchor proteins of the cytoskeleton onto the plasma membrane and the GAC is due to the incompressibility of the plasma membrane. Therefore, the dilation modulus for the GAC should be larger than that of the LAC.

\subsection{Optical tweezers test and simulation setup}

Micropipette aspiration (MA) and optical tweezers (OT) are both popular experimental techniques for the experimental measurement of RBC membrane properties. OT testing has been preferred to MA since its development because of the high resolution with which the force can be applied and the displacement measured [31]. In an OT test of a RBC, a uniaxial load is applied via two silica beads on two opposite ends of the RBC. The axial and the transverse diameters with respect to the stretching force are recorded as the output, see Fig. 12. The details of the OT test setup can be found in Dao et al. [31].

In our simulations different RBC membrane models are constructed using a network but with four spring types corresponding to a linearlyelastic (linear and truss networks), a strain-softening ( $\mathrm{NH}$ network) and

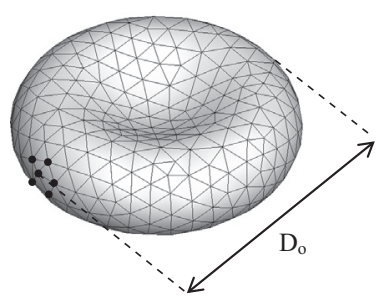

(a)

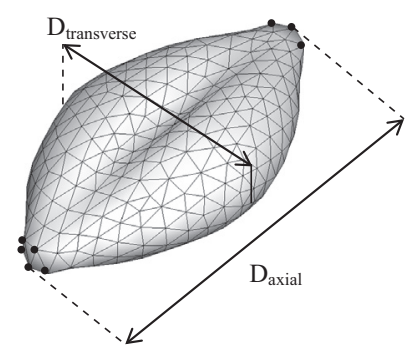

(b)
Fig. 12. Illustration of an OT test and a simulation setup. (a) A RBC in its initial state and (b) the cell under uniaxial loading. The black dots on both ends of the RBC model represent the nodes in contact with silica beads. $D_{0}$ is the initial diameter of the RBC, and $D_{\text {transverse }}$ and $\mathrm{D}_{\text {axial }}$ are the transverse and axial diameters of the deformed $\mathrm{RBC}$ respectively.

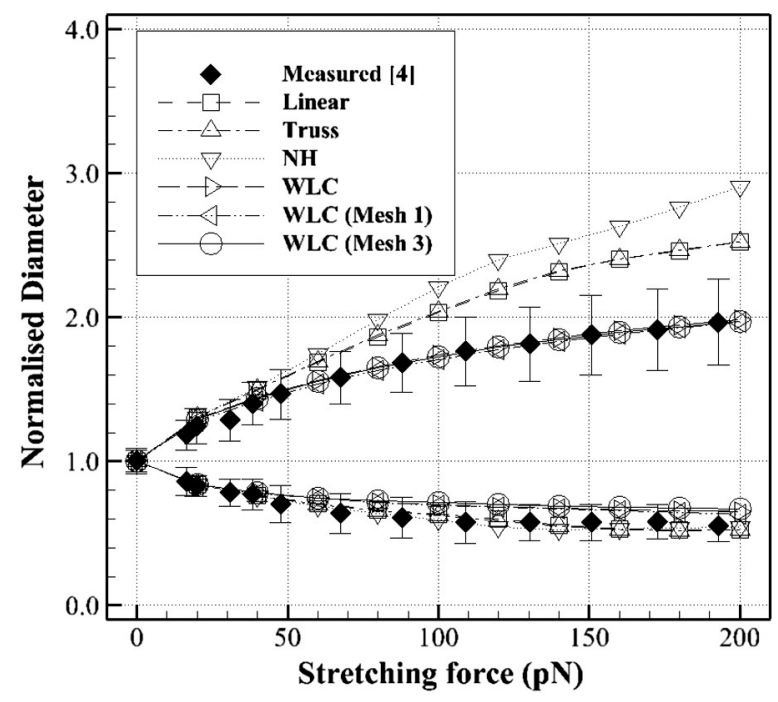

Fig. 13. Axial (upper data) and transverse (lower data) diameters of a RBC versus stretching force. The diameters are normalised by dividing by the initial RBC diameter.

a strain-hardening (WLC network) membrane. The deformation of the 3D RBC model is achieved via the position update of the network nodes as explained in Section 2.2, i.e. Eq. (2). To replicate the OT test, two equal forces are applied to opposite ends of the RBC model representing the pulling forces by the silica beads. As a silica bead [32] is $2 \mu \mathrm{m}$ in diameter, the maximum contact area between a bead and a RBC is about $2.3 \%$ of the cell surface area. In our simulations the force is equally distributed over $2 \%$ [32] of the nodes at each end of the RBC. For example, the uniaxial force is equally distributed on 26 nodes on each end of the mesh 2 RBC model as the model has 1344 nodes.

\subsection{Comparison of the predictions with experimental measurements}

Fig. 13 shows the predicted RBC diameters with respect to the stretching force in the OT test. The spring-element type employed is indicated in the legend. For the axial diameter, the linear and truss-spring networks have almost identical performance. Also, it is interesting to see that both models have three linear stretching ranges: $0-20 \mathrm{pN}$, 20-130 pN and 130-200 pN. Within these ranges, the model diameter change is nearly linear with the stretching force. The NH-spring network experiences more severe deformation. Again, three linear stretching ranges are observed: 0-20 pN, 20-110 pN and 110-200 pN. The WLC model is stiffer than the others and the simulation results fall along the centre line of the experimental measurements for the axial diameter. Importantly, the predicted results of the WLC models with different mesh densities agree well with each other which suggest that the mesh density has little effect on the membrane elasticity, a result which agrees with the observations by Fedosov et al. [32]. For the transverse diameter, the predictions compare adequately with the experimental measurements, and all the results drop within the measurement range.

Fig. 14 shows the degree of shear, i.e. $\beta$ in Eq. (18), on the discrete membrane surface when the RBC models are subjected to $100 \mathrm{pN}$ uniaxial loading. The degree of shear varies gradually throughout the membrane surface as some parts of the membrane are under uniaxial loading and some under isotropic loading. From observation of Fig. 14(a)-(d), the distribution of the degree of shear is almost the same between RBC models and the difference is due to the different degree of stretch elongations of the models. Although the OT test is a symmetric experiment, the non-symmetrical degree of shear and some local defects are observable due to the variation of local network randomness. From observation of Fig. 14(d)-(f), the symmetric and local defects of degree of shear both are influenced by the mesh density 


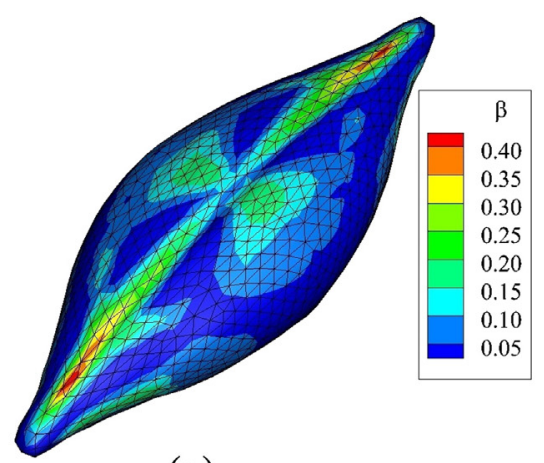

(a)

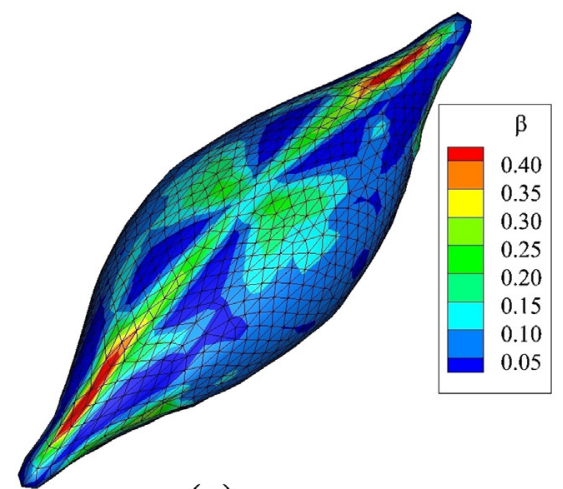

(c)

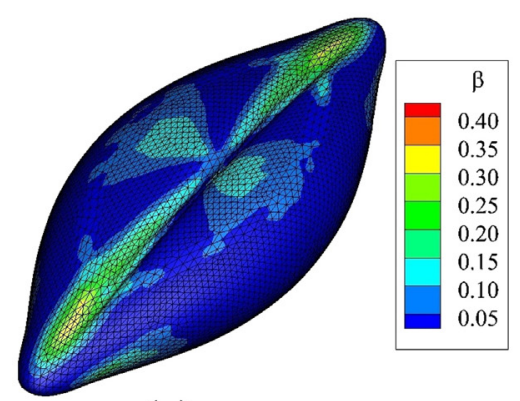

(e)

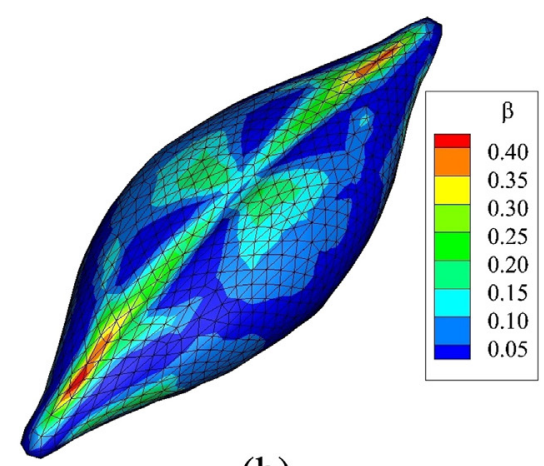

(b)

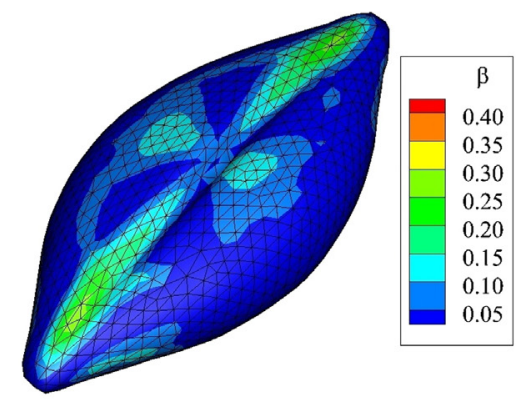

(d)

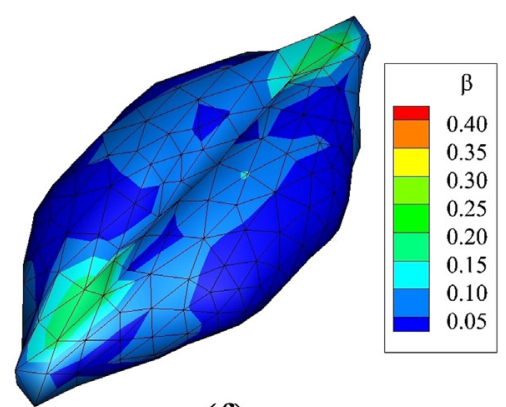

(f)

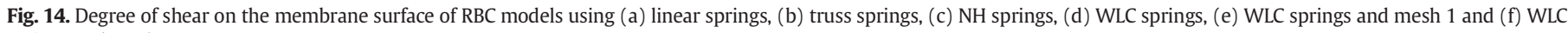
springs and Mesh 3.

of the network, e.g. a fine-mesh network leads to a detailed deformation profile and vice versa.

\section{Discussion}

In this work all spring-networks employed have approximateevenly distributed spring elements, e.g. spring-element density in one region is approximate to that of the other regions. However, a non-zero network randomness implies unevenly distributed spring elements across network surface which leads to a variation in local network mechanics. Therefore, network annealing [17] is always preferable before membrane simulation using spring networks.

A membrane model using a spring network has the advantage of a simpler mathematical formulation in comparison to a continuum membrane model. However, the exact properties of a spring network are difficult to estimate in 3D applications due to the network randomness. Currently, it is common that the in-plane properties of a spring network, e.g. shear modulus, are estimated assuming the isotropic topology [32] and results in the under-prediction of the property. For example,
Fedosov et al. [32] claimed that mesh topology affects the elasticity of the RBC models and that RBC models with higher randomness tend to be stiffer than those with lower randomness. On the other hand, a conflict occurs that a high network-randomness leads to an underprediction of network property and a low randomness leads to the direction dependence of the network elasticity. Therefore, a balance has to be maintained in numerical simulations.

In this work the primary focus is on the in-plane membrane elasticity and limited attention is given to out-of-plane bending. Currently, the bending resistance of RBC membrane models is commonly derived from the angle changes between the neighbouring triangle elements, e.g. the current RBC model. The corresponding bending modulus is estimated in the limit of small deformation [17]. Additionally, the estimation assumes the infinitesimal thickness of the RBC membrane which neglects the energy stored in the area-difference between inner and outer sides of the cell membrane and results in the under-estimation of the bending energy $[3,33]$. As a result, the shape details of the current membrane model may be influenced by the large in-plane shearing energy [11,34], e.g. the wrinkle in the centre of the RBC models in Fig. 14. 
However, the OT results are not affected since the wrinkle details have little influence on the diameters of the RBC models. In brief, the accurate representation of the membrane details of the current RBC model is limited to moderate deformation.

\section{Conclusions}

The effects of network parameters on planar-spring-network mechanics in large deformation are investigated for the first time in this work. The results indicate that the mechanical behaviour of spring-network models is conditionally equivalent to that of continuum-membrane models. In the application of spring networks for RBC modelling, it is found that the findings for the planar-springnetworks mechanics are transferable to membrane modelling using spring networks in 3D.

The elastic behaviour of RBC models using different spring-element types are compared for the first time in this work. It has been shown that a discrete RBC model, which uses a spring network to represent the cell membrane, accurately captures the elastic behaviour of a RBC in the OT test. Therefore, we may conclude, contradicting the conclusion made by Omori et al. [18], that a spring network with a surface constraint is sufficient for modelling the RBC membrane. Additionally, an unstructured network with certain randomness is desirable for a directional independence of network elasticity in large deformation. Finally, in order to capture the strain-hardening nature of the biological membrane, hardening spring-element types must be employed. The employment of linear and softening spring-element types lead to over-estimation of membrane shear modulus, e.g. [30,35].

\section{Acknowledgement}

This work has been supported by the Fiosraigh Research Scholarship provided by the Dublin Institute of Technology.

\section{Appendix A}

Consider the deformation of an isotropic linear-spring network, in which the network may be considered as a combination of a large number of identical equilateral triangles. The network is regular and the deformation upon external loading is also uniformly distributed. Therefore, for simplicity, a triangle may be extracted from the network to represent the network deformation as shown in Fig. 15. The triangle

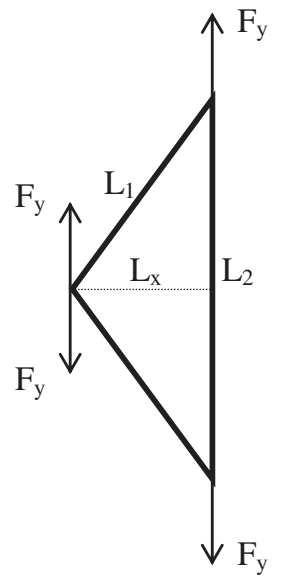

(a)

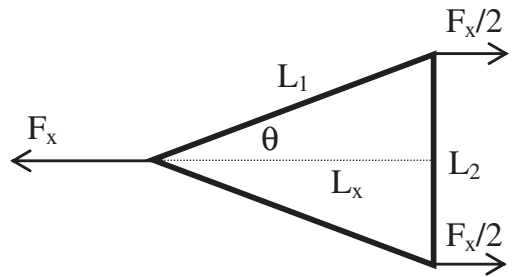

(b)
Fig. 15. Illustration of two deformation scenarios of a spring triangle. The vertical deformation (a) is caused by the vertical loading, i.e. $F_{x}=0$ and $F_{y} \neq 0$, and the horizontal deformation (b) is caused by the horizontal loading, i.e. $\mathrm{F}_{\mathrm{x}} \neq 0$ and $\mathrm{F}_{\mathrm{y}}=0 . \mathrm{L}_{1}$ and $\mathrm{L}_{2}$ are the instantaneous lengths of springs 1 and 2 respectively, $L_{x}$ is the horizontal component of $\mathrm{L}_{1}$ and $\theta$ is the instantaneous angle between spring 1 and the horizontal direction. is formed by three linear-spring elements with initial lengths of $\mathrm{L}$ and shear modulus of $(\sqrt{3} / 4) \mathrm{k}$ [36], where $\mathrm{k}$ is the spring constant.

The triangle deformation is caused by the tensile forces, i.e. $\mathrm{F}_{\mathrm{x}}$ and $\mathrm{F}_{\mathrm{y}}$, in principal directions. In the vertical deformation, i.e. $\mathrm{F}_{\mathrm{x}}=0$ and $\mathrm{F}_{\mathrm{y}} \neq 0$, in Fig. 15(a), the length of spring 1 remains constant until spring 1 is in-line with spring 2 . Therefore, the stretch-strain relationship in the vertical direction is

$F_{y}=k\left(L_{2}-L\right)=k\left(\lambda_{2}-1\right) L$

where $\lambda_{2}$ is the stretch ratio of the spring 2, i.e. $\lambda_{2}=L_{2} / L$. Since spring 1 is not stretched, then from Pythagoras' theorem we have

$L_{x}=\sqrt{L_{1}^{2}-\left(\frac{L_{2}}{2}\right)^{2}}=\sqrt{L_{1}^{2}-\left(\frac{\lambda_{2}}{2} L\right)^{2}}=\frac{L}{2} \sqrt{4-\lambda_{2}^{2}}$.

Therefore, the dimensionless tension is

$\frac{T_{y}}{\mu_{o}}=\frac{F_{y}}{\mu_{o} L_{x}}=\frac{k\left(\lambda_{2}-1\right) L}{\left(\frac{L}{2} \sqrt{4-\lambda_{2}^{2}}\right)\left(\frac{\sqrt{3}}{4} k\right)}=\frac{8}{\sqrt{3}} \frac{\lambda_{2}-1}{\sqrt{4-\lambda_{2}^{2}}}$.

Also the vertical strain is

$\varepsilon_{2}=\frac{1}{2}\left(\lambda_{2}^{2}-1\right)$

In the horizontal deformation, i.e. $\mathrm{F}_{\mathrm{x}} \neq 0$ and $\mathrm{F}_{\mathrm{y}}=0$, in Fig. 15(b), spring 1 is stretched due to the horizontal loading and spring 2 is compressed due to the vertical stretching component of spring 1, i.e.

$\mathrm{F}_{x}=2 \mathrm{k}\left(\mathrm{L}_{1}-\mathrm{L}\right) \cos \theta$

$\mathrm{k}\left(\mathrm{L}-\mathrm{L}_{2}\right)=2 \mathrm{k}\left(\mathrm{L}_{1}-\mathrm{L}\right) \sin \theta$.

Also from Pythagoras' theorem we have

$\frac{\mathrm{L}_{2}}{2 \mathrm{~L}_{1}}=\sin \theta$

Substituting Eqs. (31) into (30) to eliminate $\mathrm{L}_{1}$ gives

$\mathrm{k}\left(\mathrm{L}-\mathrm{L}_{2}\right)=2 \mathrm{k}\left(\frac{\mathrm{L}_{2}}{2 \sin \theta}-\mathrm{L}\right) \sin \theta=2 \mathrm{k}\left(\frac{\mathrm{L}_{2}}{2}-\mathrm{L} \sin \theta\right)$.

Rearranging Eq. (32) we get

$\mathrm{L}_{2}=\mathrm{L}\left(\frac{1}{2}+\sin \theta\right)$

Next, substituting Eqs. (31) and (33) into Eq. (29) to eliminate $\mathrm{L}_{1}$ and $\mathrm{L}_{2}$ gives

$\mathrm{F}_{\mathrm{x}}=2 \mathrm{~kL}\left(\frac{\frac{1}{2}+\sin \theta}{2 \sin \theta}-1\right) \cos \theta$. 
Therefore, the dimensionless tension on the triangle is

$\frac{\mathrm{T}_{\mathrm{x}}}{\mu_{0}}=\frac{\mathrm{F}_{\mathrm{x}}}{\mu_{0} \mathrm{~L}_{2}}=\frac{2 \mathrm{~kL}\left(\frac{\frac{1}{2}+\sin \theta}{2 \sin \theta}-1\right) \cos \theta}{\left(\frac{\sqrt{3}}{4} \mathrm{k}\right)\left(\mathrm{L}\left(\frac{1}{2}+\sin \theta\right)\right)}=\frac{8}{\sqrt{3}}\left(\frac{1}{2 \sin \theta}-\frac{1}{\frac{1}{2}+\sin \theta}\right) \cos \theta$.

\section{Also the horizontal stretch is}

$\lambda_{\mathrm{x}}=\frac{\mathrm{L}_{\mathrm{x}}}{\frac{\sqrt{3}}{2} \mathrm{~L}}=\frac{\frac{\mathrm{L}_{2}}{2}}{\left(\frac{\sqrt{3}}{2} \mathrm{~L}\right)(\tan \theta)}=\frac{\mathrm{L}\left(\frac{1}{2}+\sin \theta\right)}{\sqrt{3} \mathrm{~L} \tan \theta}=\frac{\frac{1}{2}+\sin \theta}{\sqrt{3} \tan \theta}$.

\section{Therefore the horizontal strain is}

$\varepsilon_{\mathrm{x}}=\frac{1}{2}\left(\lambda_{x}^{2}-1\right)=\frac{1}{2}\left(\left(\frac{\frac{1}{2}+\sin \theta}{\sqrt{3} \tan \theta}\right)^{2}-1\right)$.

\section{References}

[1] R. Skalak, A. Tozeren, R.P. Zarda, S. Chien, Biophys. J. 13 (1973) 245.

[2] E.A. Evans, Biophys. J. 13 (1973) 926.

[3] G. Gompper, M. Schick, Soft Matter: Lipid Bilayers and Red Blood Cells, Wiley-VCH, John Wiley distributor, Weinheim Chichester, 2008. 254.

[4] J.P. Mills, L. Qie, M. Dao, C.T. Lim, S. Suresh, Mech. Chem. Biosyst. 1 (2004) 169.

[5] T. Moschandreou (Ed.), Blood Cell - An Overview of Studies in Hematology, InTech, 2012.

[6] C. Pozrikidis, Ann. Biomed. Eng. 31 (2003) 1194.
[7] C. Pozrikidis, J. Fluid Mech. 297 (1995) 123.

[8] J.F. Gross, A. Popel, Mathematics of Microcirculation Phenomena, Raven Press, New York, 1980. (p. xi, 174 pp.).

[9] S. Suresh, J. Spatz, J.P. Mills, A. Micoulet, M. Dao, C.T. Lim, M. Beil, T. Seufferlein, Acta Biomater. 1 (2005) 15.

[10] D.E. Discher, D.H. Boal, S.K. Boey, Biophys. J. 75 (1998) 1584.

[11] J. Li, M. Dao, C.T. Lim, S. Suresh, Biophys. J. 88 (2005) 3707.

[12] D.A. Fedosov, B. Caswell, G.E. Karniadakis, Conf. Proc. 31st Annu. Int. Conf. IEEE Eng. Med. Biol. Soc, 2009, pp. 4266-4269.

[13] W. Pan, D.A. Fedosov, B. Caswell, G.E. Karniadakis, Microvasc. Res. 82 (2011) 163.

[14] M. Dupin, I. Halliday, C. Care, L. Alboul, L. Munn, Phys. Rev. E. 75 (2007) 1.

[15] P. Bagchi, P.C. Johnson, A.S. Popel, J. Biomech. Eng. 127 (2005) 1070.

[16] T.W. Secomb, Med. Eng. Phys. 33 (2011) 800.

[17] C. Pozrikidis, Computational Hydrodynamics of Capsules and Biological Cells, CRC Press, Boca Raton, 2010. (p. xv, 311 pp.)

[18] T. Omori, T. Ishikawa, D. Barthès-Biesel, A.-V. Salsac, J. Walter, Y. Imai, T. Yamaguchi, Phys. Rev. E. 83 (2011) 1

[19] A. Van Gelder, J. Graph. Tools 3 (1998) 21.

[20] J.C. Hansen, R. Skalak, S. Chien, A. Hoger, Biophys. J. 70 (1996) 146.

[21] J.C. Hansen, R. Skalak, S. Chien, A. Hoger, Biophys. J. 72 (1997) 2369.

[22] H. Delingette, IEEE Trans. Vis. Comput. Graph. 14 (2008) 329.

[23] H. Delingette, in: F. Bello, P.J.E. Edwards (Eds.), Biomed. Simul, Springer Berlin, Heidelberg, 2008, pp. 40-48.

[24] M.M. Dupin, I. Halliday, C.M. Care, L.L. Munn, Int. J. Comput. Fluid Dyn. 22 (2008) 481.

[25] D. Barthès-Biesel, A. Diaz, E. Dhenin, J. Fluid Mech. 460 (2002) 211.

[26] R. Afrin, M. Nakaji, H. Sekiguchi, D. Lee, K. Kishimoto, A. Ikai, Cytoskeleton (Hoboken) 69 (101) (2012).

[27] M. Dao, J. Li, S. Suresh, Mater. Sci. Eng. C 26 (2006) 1232.

[28] E.A. Evans, R. Skalak, Mechanics and Thermodynamics of Biomechanics, CRC Press, Boca Raton, 1980. 254.

[29] E.A. Evans, Y.-C. Fung, Microvasc. Res. 4 (1972) 335.

[30] Y. Imai, H. Kondo, T. Ishikawa, C. Teck Lim, T. Yamaguchi, J. Biomech. 43 (2010) 1386.

[31] M. Dao, C.T. Lim, S. Suresh, J. Mech. Phys. Solid 51 (2003) 2259.

[32] D.A. Fedosov, B. Caswell, G.E. Karniadakis, Comput. Methods Appl. Mech. Eng. 199 (2010) 1937.

[33] T. Klöppel, W.A. Wall, Biomech. Model. Mechanobiol. 445 (2011).

[34] D. Barthès-Biesel, Compt. Rendus Phys. 10 (2009) 764.

[35] C.T. Lim, M. Dao, S. Suresh, C.H. Sow, K.T. Chew, Acta Mater. 52 (2004) 1837.

[36] D.H. Boal, Mechanics of the Cell, Second ed., 2002. 608 (Cambridge). 